\title{
Aspects Relating of the Oxidative Stress to Living Organisms
}

\section{Monica Butnariu ${ }^{1 *}$ and lonel Samfira ${ }^{2}$}

${ }^{1}$ Discipline of Chemistry and Vegetal Biochemistry, Banat's University of Agricultural Sciences and Veterinary Medicine from Timisoara, Romania ${ }^{2}$ Discipline of Grassland and Fodder plants culture, Banat's University of Agricultural Sciences and Veterinary Medicine from Timisoara, Romania

Free radicals (pro-oxidants) are compounds belonging to the radical groups; they are active biochemically and biologically, and they destroy cell membranes, nuclei, and cytoplasm, producing and maintaining an intense oxidative stress. This is a consequence of the presence of one or several free electrons on the last layer of an atom in the molecule. Free radicals are oxygenated electron-deficit anions that do not make up salts, acids, or bases, but keep this reactive (free) form [1].

Oxygen electronic structure explains why the element, though a free diradical (two unpaired electrons) have low reactivity. Oxygen is essential for aerobic bodies' life, but, in concentrations that are too high, it can turn toxic. Molecular oxygen in fundamental state is inert, and its partial reduction results in active oxygen species-the most dangerous free radicals [2]. This group of compounds is called Reactive Oxygen Species (ROS). ROS is a term including not only oxygen radicals (O and $\mathrm{H}$ ), but also oxygen non-radicalic derivatives, including hydrogen peroxide $\left(\mathrm{H}_{2} \mathrm{O}_{2}\right)$, hypochlorous acid $(\mathrm{HOCl})$, and ozone $\left(\mathrm{O}_{3}\right)$ [3. Even if ROS are not true free radicals, these species are molecule reactive [4].

Under physiological conditions, ROS are produced in small amounts in the cells [5.

The interaction between these species and lipid membranes, nucleic acids, proteins and enzymes or other small molecules, leads inevitably to cell lesions (Figure 1).

These cell lesions are one of the factors leading to ageing and degenerative diseases at cell level, there are several types of reactive species.

The variety of free radicals in nature resulted from different processes (ultraviolet radiations, gamma radiations, action of specific particles, etc.) makes their classification difficult $[6,7]$.

From the point of view of the nature of the element containing free electrons, free radicals can be: superoxide, peroxide, hydroxide, nitric oxide, nitrite, nitrate, alkoxyl (Table 1).

Cell production of ROS roots in enzymatic and non-enzymatic sources.

Electron transfer from proteins or enzymatic systems can lead to ROS as a result of electron transfer reactions. This "unintentional" generation of ROS in mitochondria represents $1-2 \%$ of the total $\mathrm{O}_{2}$ consumed in reducing conditions. Body oxygen content represents $65 \%$ and inhaled air oxygen content is $21 \%$. Cells generate aerobic energy, reducing $\mathrm{O}_{2}$ to water.

Oxygen can be used in catabolic and anabolic processes, allowing larger amounts of energy than possible in its absence [8].

Oxygen has a particular electronic structure in its fundamental state, with two non-participating electrons on the last layer, each of which is localised on an orbital $n^{\star}$ (Figure 2).

These two electrons have the same quantum number of spin; thus, if $\mathrm{O}_{2}$ tries to oxidate a compound by accepting two electrons, they need to have a parallel spin number to occupy the free spaces in the orbitals $n^{\star}$ (in an orbital, two electrons have anti-parallel spins $+1 / 2$ and $-1 / 2$ ) [9].

This particularity asks for a restriction of oxidations, determining higher or lower reactivity, depending on the nature of the electron donor (Table 2).

All oxidations in nature are based on these two pathways, even if the forms may seem varied.

Peroxides and superoxides are anions that have oxidative action (peroxides, alkoxyls, nitrosamines, acrolein) derive from $\mathrm{H}_{2} \mathrm{O}_{2}$ and from other sources/processes, like the anions resulted from the degradation (rancidisation, proteolytic degradation, etc.) of lipid-rich foods (lipid peroxides, lipid alkoxyls) or from food processing (frying, refreezing, etc.).

Hypochlorous acid behaves like free radicals (but they do not belong to this class).

Other sources of free radicals are nitrosamines and unsaturated aldehydes (acrolein), very active and destructive because of their action on cell membranes [10].

Evolutively, nature has selected and included in the composition of the organisms, reactions generating free radicals with multiple roles: functional, intracellular communication, or destructive, cytolitic. If, at molecular level, the main target of the free radicals is the free or protein groups $\mathrm{SH}$, at cellular level, the major goal is cell membranes.

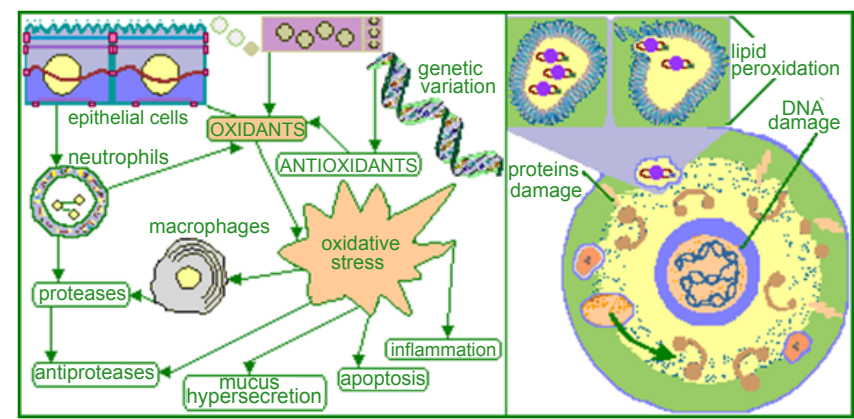

Figure 1: Effects of free radicals.

*Corresponding author: Monica Butnariu, Discipline of Chemistry and Vegetal Biochemistry, Banat's University of Agricultural Sciences and Veterinary Medicine from Timisoara, 300645, Calea Aradului 119, Timis, Romania, Tel: +40-0-256-277464; E-mail: monicabutnariu@yahoo.com, monica_butnariu@usab-tm.ro

Received July 20, 2013; Accepted July 22, 2013; Published July 22, 2013

Citation: Butnariu M, Samfira I (2013) Aspects Relating of the Oxidative Stress to Living Organisms. J Biodivers Endanger Species 1: e106. doi:10.4172/ 2332-2543.1000e106

Copyright: @ 2013 Butnariu M. et al, This is an open-access article distributed under the terms of the Creative Commons Attribution License, which permits unrestricted use, distribution, and reproduction in any medium, provided the original author and source are credited. 
Citation: Butnariu M, Samfira I (2013) Aspects Relating of the Oxidative Stress to Living Organisms. J Biodivers Endanger Species 1: e106. doi:10.4172/2332-2543.1000e106

Page 2 of 2

\begin{tabular}{|c|c|}
\hline Species/Common Name & Systematic Name/Alternative and Comments \\
\hline CO/carbon monoxide & carbon monoxide/Oxidomethanediyl $\left(\mathrm{CH}_{3}^{\circ}\right)$ \\
\hline $\mathrm{CO}_{2} /$ carbon dioxide & carbon dioxide/dioxidomethane \\
\hline $\mathrm{CO}_{2}^{-}$//carbon dioxide radical anion & $\begin{array}{l}\text { dioxidocarbonate }(\cdot 1-) / \text { oxidooxomethyl } \\
\text { radical }\end{array}$ \\
\hline $\mathrm{CO}_{3}^{-}$-/carbonate radical & trioxidocarbonate $(\circ 1-)$ \\
\hline $\mathrm{H} \circ /$ hydrogen atom & monohydrogen $\left({ }^{\circ}\right)$ \\
\hline $\mathrm{H}_{2} \mathrm{O} /$ water & dihydrogen monoxide/oxidane \\
\hline $\mathrm{H}_{2} \mathrm{O}_{2}$ /hydrogen peroxide & dihybridodioxide/dioxidane \\
\hline $\mathrm{H}_{3} \mathrm{C}$ 。 & methyl radical \\
\hline $\mathrm{HNO}_{2}$ & nitrous acid \\
\hline $\mathrm{HNO}_{3}$ & nitric acid \\
\hline $\mathrm{HO} /$ /hydroxyl radical & hydridooxygen/oxidanyl \\
\hline $\mathrm{HO}_{2}^{-/}$hydridodioxygen (1-) & $\begin{array}{l}\text { dioxidanide, hydrogendioxide (1-)/ } \\
\text { hydrogenperoxide (1-) }\end{array}$ \\
\hline $\mathrm{HO}_{2}$ /hydroperoxyl, but is obsolete & $\begin{array}{l}\text { Hydridodioxygen }\left({ }^{\circ}\right) \text { dioxidonyl/ } \\
\text { hydrodioxyl, perhydroxyl }\end{array}$ \\
\hline $\mathrm{HO}_{3}$ /hydrogen trioxide radical & hydridotrioxygen $\left({ }^{\circ}\right) /$ trioxidanyl \\
\hline $\mathrm{HOCO}$ 。 & hydroxidooxidocarbon $\left({ }^{\circ}\right)$ \\
\hline $\mathrm{HOCO}_{2}$ & hydroxidodioxidocarbon $\left({ }^{\circ}\right)$ \\
\hline $\mathrm{HOCl} /$ hypochlorous acid & hydrogenoxidochlorate \\
\hline HOBr/hypobromous acid & hydrogenoxidobromate \\
\hline $\mathrm{HOI} /$ hypoiodous acid & hydrogenoxidoiodate \\
\hline HOSCN/hypothiocyanous acid & hydrogenoxidothiocyanate \\
\hline $\mathrm{HON}_{2}^{\circ}$ & $\begin{array}{l}\text { hydroxidonitrogen }\left(2^{\circ}\right) \text { (triplet)/hydrogen } \\
\text { oxidonitrate }(2 \circ)\end{array}$ \\
\hline HOOCO/ (hydridodioxido) & oxidocarbon $(\circ)$ \\
\hline HOONO/peroxynitrous acid & $\begin{array}{l}\text { hydrogenoxidoperoxidonitrate/ } \\
\text { nitrosodioxidane }\end{array}$ \\
\hline$(\mathrm{NO})_{2}^{{ }^{-}}$ & bis (oxidonitrate) $(n-n)(\circ 1-)$ \\
\hline $\mathrm{N}_{2} \mathrm{O} /$ nitrous oxide & dinitrogen monoxide \\
\hline $\mathrm{N}_{2} \mathrm{O}^{-} 。$ & oxidodinitrate $(\bullet 1-)$ \\
\hline $\mathrm{N}_{2} \mathrm{O}_{3}$ & dinitrogen trioxide \\
\hline $\mathrm{N}_{3}$ /azidyl radical & trinitrogen $(2 n-n)\left({ }^{\circ}\right)$ \\
\hline $\mathrm{NO} /$ nitric oxide, but is obsolete & $\begin{array}{l}\text { oxidonitrogen }(\circ) / \text { oxoazanyl, nitrogen } \\
\text { monoxide }\end{array}$ \\
\hline $\mathrm{NO}^{-}\left(2^{\circ}\right) /$ nitroxyl & oxidonitrate $(2 \cdot 1-)$ (triplet) \\
\hline $\mathrm{NO}_{2}-/$ nitrite & dioxidonitrate (1-) \\
\hline $\mathrm{NO}_{2} \circ /$ nitrogen dioxide & dioxidonitrogen \\
\hline $\mathrm{NO}_{2}^{2-0}$ & dioxidonitrate $(\cdot 2-)$ \\
\hline $\mathrm{NO}^{3-} /$ nitrate & trioxidonitrate (-) \\
\hline $\mathrm{NO}_{3} \circ /$ nitrogen trioxide & trioxidonitrogen $(\circ) /$ nitrosoxidanyl \\
\hline $\mathrm{NO}_{3}{ }^{2-\circ}$ & trioxidonitrate $\left({ }^{\circ}-\right)$ \\
\hline O-/radical anion of $\mathrm{HO}$ 。 & oxide $(\circ 1-) /$ oxidanidyl \\
\hline $\mathrm{O}_{2} 0^{-} /$superoxide & dioxide $(\circ 1-) /$ dioxidanidyl \\
\hline $\mathrm{O}_{2}^{+} \mathrm{ol}$ & dioxygen $(\circ 1+)$ \\
\hline $\mathrm{O}_{2}^{2}{ }^{2} /$ oxygen, usually $\mathrm{O}$ written $\mathrm{O}_{2}$ & dioxygen (triplet)/dioxidanediyl \\
\hline $\mathrm{O}_{3}$ /ozone & trioxygen \\
\hline $\mathrm{O}_{3}{ }^{\circ}$ /ozonide & trioxide $(\circ 1-) /$ trioxidanidyl \\
\hline OCl-/hypochlorite & oxidochlorate (1-) \\
\hline OBr-/hypobromite & oxidobromate (1-) \\
\hline Ol-/hypoiodite & oxidoiodate (1-) \\
\hline OSCN-/hypothiocyanate & oxidothiocyanate (1-) \\
\hline $\mathrm{OCOO}^{-}$ & (dioxido) oxidocarbonate $(\bullet 1-)$ \\
\hline ONOO-/peroxynitrite & oxidoperoxidonitrate (1-)/nitrosodioxidanide \\
\hline $\mathrm{ONOOH} /$ peroxynitrous acid & hydrogen-oxidoperoxinitrate/nitrosodioxidane \\
\hline ONOO。 & $\begin{array}{l}\text { (dioxido)oxidonitrogen }\left({ }^{\circ}\right) / \\
\text { nitrosodioxidanyl }\end{array}$ \\
\hline
\end{tabular}

\begin{tabular}{|c|c|c|c|c|}
\hline \multicolumn{5}{|c|}{ Reactive oxygenspecies (-unpaired electrons) } \\
\hline $\begin{array}{l}\text { Ö::Ö } \\
\text { Oxygen } \\
\text { O2 }\end{array}$ & Superoxide anion & $\begin{array}{c}\text {-O:-:O" } \\
\text { Peroxide } \\
\text { O2-2 }\end{array}$ & $\mid \begin{array}{c}\text { Hydroxyl radical } \\
\text {-OH }\end{array}$ & $\begin{array}{l}\text { Hydroxyl ion } \\
\mathrm{OH}^{-0} \mathrm{H}\end{array}$ \\
\hline
\end{tabular}

Figure 2: Reactive oxygen species

No. Species/Abbreviation Name

\begin{tabular}{|l|l|}
\hline Asc; $\mathrm{AscH}^{-} ; \mathrm{Asc}^{-}$ & $\begin{array}{l}\text { ascorbate, general; ascorbate monoanion; ascorbate } \\
\text { radical }\end{array}$ \\
\hline CAT & catalase \\
\hline HRP & peroxidase \\
\hline GPx & glutathione peroxidase \\
\hline GR & $\begin{array}{l}\text { glutathione disulfide reductase; often referred to as } \\
\text { glutathione reductase }\end{array}$ \\
\hline Grx & glutaredoxin \\
\hline GSH & glutathione, not reduced glutathione (a misnomer) \\
\hline GST & glutathione S transferase \\
\hline LDL & low density lipoprotein \\
\hline OH- & hydroxide anion, not to be confused with HO \\
\hline PUFA & polyunsaturated fatty acid \\
\hline RO $\circ$ & alkoxyl radical; not alkoxy \\
\hline ROO & alkyl dioxygen $(\circ)$, alkyldioxyl, alkylperoxyl radical; \\
\hline SOD & not peroxy \\
\hline
\end{tabular}

*These are commonly used abbreviations. Others appear in the literature.

Table 2: Common Abbreviations.

\section{References}

1. Veda S, Platel K, Srinivasan K (2008) Influence of food acidulants and antioxidant spices on the bioaccessibility of beta-carotene from selected vegetables. J Agr Food Chem 56: 8714-8719.

2. Cynshi O, Tamura K, Niki E (2010) Design, synthesis, and action of antiatherogenic antioxidants. Method Mol Biol 610: 91-107.

3. Takabe W, Li R, Ai L, Yu F, Berliner JA, et al. (2010) Oxidized Low -Density Lipoprotein -Activated c -Jun $\mathrm{NH}_{2}$-Terminal Kinase Regulates Manganese Superoxide Dismutase Ubiquitination: Implication for Mitochondrial Redox Status and Apoptosis. Arterioscl Throm Vas 30: 436-441.

4. Dadali VA, Tutel'ian VA, Dadali luV, Kravchenko LV (2010) Carotenoids. Bioavailability, biotransformation, antioxidant properties. Vop pitaniia 79: 4-18.

5. Surapaneni KM, Venkataramana G (2007) Status of lipid peroxidation, glutathione, ascorbic acid, vitamin $\mathrm{E}$ and antioxidant enzymes in patients with osteoarthritis. Indian Journal of Med Sci 61: 9-14.

6. Kanamoto T, Rimayanti UHO, Kiuchi Y (2011) Platelet -derived growth factor receptor alpha is associated with oxidative stress -induced retinal cell death. Curr Eye Res 36: 336-40.

7. Pattillo CB, Pardue S, Shen X, Fang K, Langston W, et al. (2010) ICAM -1 cytoplasmic tail regulates endothelial glutathione synthesis through a NOX $\mathrm{N}_{4} / \mathrm{PI}_{3}$ -kinase -dependent pathway. Free Radical Bio Med 49: 1119-1128.

8. Jacobs AC, Resendiz MJ, Greenberg MM (2011) Product and mechanistic analysis of the reactivity of a C6 -pyrimidine radical in RNA. J Am Chem Soc 133: 5152-5159.

9. Pickrell AM, Fukui H, Moraes CT (2009) The role of cytochrome c oxidase deficiency in ROS and amyloid plaque formation. J Bioenerg Biomembr 41 : 453-456.

10. Rizzo AM, Berselli P, Zava S, Montorfano G, Negroni M, et al. (2011) Endogenous Antioxidants and Radical Scavengers. Adv Exp Med Biol 698 52-67.

Table 1: Formulae and IUPAC Recommended Names of Simple Compounds Containing $\mathrm{C}, \mathrm{H}$, and $\mathrm{O}$ in Free Radical Biology 